\title{
Single-Event Dynamics of High-Performance HBTs and GaAs MESFETs
}

\author{
Dale McMorrow, Todd Weatherford, ${ }^{\dagger}$ Alvin R. Knudson, ${ }^{\dagger}$ Lan Hu Tran, ${ }^{\dagger}$ \\ Joseph S. Melinger, and Arthur B. Campbell, \\ Naval Research Laboratory, Code 6613, Washington D.C. 20375
}

\begin{abstract}
Picosecond charge-collection transients measured for GaAs/AlGaAs HBTs following $3.0 \mathrm{MeV}$ $\alpha$-particle and $620 \mathrm{~nm}$ picosecond laser excitation reveal charge-collection efficiencies up to twenty-eight times smaller than for $\mathrm{GaAs}$ MESFETs, with $\sim 90 \%$ of the charge collected within $75 \mathrm{ps}$ of the ionizing event. The small charge-collection efficiency of the HBTs is a consequence of the ultrafast charge-collection dynamics in these devices. We show that picosecond laser excitation reproduces nicely the ioninduced transients, providing a valuable tool for the investigation of charge-collection and SEU phenomena in these devices.
\end{abstract}

\section{INTRODUCTION}

GaAs MESFET ICs exhibit desirable characteristics for high total dose and high dose rate environments. However, high performance GaAs MESFET ICs are susceptible to single event upset (SEU) effects [1-3], exhibiting error rates that are unacceptable for present space applications. Heterojunction bipolar technology now offers a viable alternative to MESFET technology for high performance applications. The charge collection and SEU characteristics of the present HBT technology, however, have not been fully examined.

In this paper we present ion- and laserinduced picosecond resolution charge-collection measurements of state-of-the-art AlGaAs/GaAs heterojunction bipolar transistors (HBTs). The results are compared to analogous transients measured for GaAs MESFETs. It is found that the charge collection efficiency of the HBT devices is significantly smaller than that of the MESFETs. Time resolved measurements reveal that, for the HBT devices, effectively all of the charge is collected within 250 ps of the ionizing event, in contrast to the behavior of $\mathrm{GaAs}$ MESFETs, which can exhibit transients that persist for nanoseconds [4,5] and even microseconds [6]. The absence of slower charge collection mechanisms in the HBT devices reduces significantly the total charge collected when compared to MESFETs, and suggests that circuits based on HBT technolgy will exhibit
SEU characteristics superior to those of $\mathrm{GaAs}$ MESFETs.

\section{BACKGROUND}

In recent years there have been several investigations into the mechanisms of charge collection in GaAs MESFETs [1,2,4-8]. Numerous experiments have revealed that GaAs MESFETs can exhibit charge collection in excess of that deposited by the ion (or laser pulse) $[2,6,7,9]$, and it has been suggested that this excess charge collection may be responsible for the high susceptibility of MESFETs to SEU phenomena. Much recent work has focused on the origin of this excess charge collection. Two primary mechanisms have been suggested: i) a backchannel turn-on mechanism; and ii) a bipolar source-drain mechanism. In both cases a source-drain current pathway is created, permitting electrons to be supplied by the source (via the power supply), resulting in the possibility that more charge is collected at the drain than is deposited by the ion or optical pulse.

Recent 2-D computer simulations and picosecond transient measurements $[4,5,8]$ reveal that the total amount of charge collected in buried p-layer GaAs MESFETs is determined to a large extent by the slower charge-collection pathways that can persist for nanoseconds and even microseconds. These slower mechanisms depend in a sensitive fashion on the device bias conditions, and can become significant when the device is biased near pinch off (slightly "on"). Both experiment and simulation results suggest that the excess hole concentration created by the ion in the substrate and buried p-layer induces a loss of gate control in the device (the backchannel turn on mechanism noted above), giving rise to significant source-drain currents that persist for the lifetime of this excess hole population. This is a field-effect phenomenon that is intrinsic to FET devices. The existence of a bipolar source-drain gain mechanism is also evident in both experimental $[1,7]$ and simulation [8] results. In the simulations, however, this effect is significant only for tens of picoseconds following the ion strike, and appears to be a minor contributor to the charge enhance- 
ment in the buried p-layer MESFETs.

A schematic diagram of an AlGaAs/GaAs HBT is illustrated in Fig. 1. We have investigated the charge collection dynamics for HBT devices from three different vendors. The structure illustrated in fig. 1 is representative of the

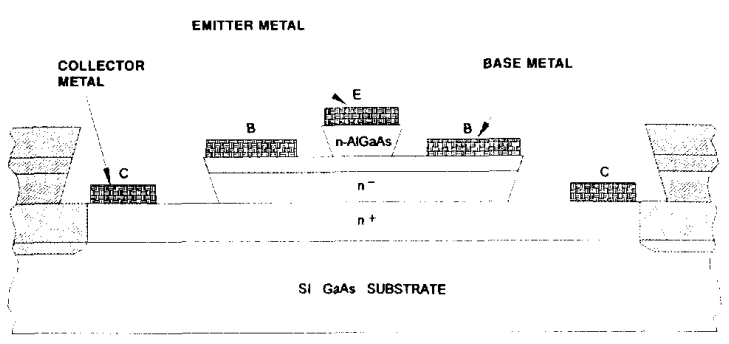

Figure 1. Schematic diagram of $\mathrm{AlGaAs} / \mathrm{GaAs}$ HB'T representative of the current technology.

current technology, however the precise structures of the devices used in this study are proprietary.

The n-p-n HBT is composed of a wide bandgap emitter (typically AlGaAs), a $\mathrm{p}^{+}$doped GaAs base, and an $\mathrm{n}^{-}$doped GaAs collector. The HBT operates similarly to a homojunction bipolar transistor in that forward bias of the base-emitter junction injects electrons (minority carriers) into the base region,. with these electrons being transported across the basecollector junction. The homojunction bipolar transistor requires a high emitter-to-base doping ratio to optimize emitter efficiency (the ratio of the efficiency of injecting electrons into the base to the efficiency of injecting holes from the base into the emitter). The HBT uses the difference of the AlGaAs and GaAs bandgaps to improve emitter efficiency. The barrier in the valence band prevents a reverse hole current into the emitter when the base-emitter junction is forward biased, while only a small barrier is seen by electrons flowing from emitter to base. This superior emitter efficiency directly improves the current gain of the device and permits a highly doped base region which results in reduced base resistance. Additionally, the fabrication techniques of the HBTs (MBE and MOCVD) result in very thin base regions (on the order of 200 angstroms) giving rise to short base transit times and high $f_{\mathrm{T}}$ (where $f_{\mathrm{T}}$ is the maximum frequency of current gain).

The differences in carrier transport between GaAs MESFETs and HBTs are significant. The most obvious is that HBTs exhibit vertical charge transport, whereas MESFETs are lateral transport devices. Transistor action in an HBT is controlled by the injection of electrons into the base region (i.e., base-emitter biasing); the MESFET is controlled by the modulation of the depletion region in a doped channel. Thus, charge deposited in the substrate below the active region of the device, which has been shown to have a large effect in MESFETs, is not expected to have a significant effect on charge collection in HBTs. This point will be elaborated on in later sections.

Some previous charge-collection work on GaAs HBTs has been performed [10,11]. Consistent with that work, the HBTs of this study exhibit significantly smaller charge-collection efficiencies than GaAs MESFETs. Picosecond laser experiments also have been performed on an InP/GaInAs/InP HBT [12]. As in the present study, ultrafast photocurrent transients were observed. The grounded-collector configuration used in that study, however, prevents a direct comparison with the present results

Related results on complementary $\mathrm{AlGaAs/}$ GaAs heterojunction insulated gate FETs (CHIGFETs) exhibit reduced charge collection and improved SEU performance over MESFETs [13]. That work suggested that the enhanced charge collection mechanisms present in MESFETs are reduced in the C-HIGFET technology. Very recently, an extensive study on a C-HIGFET SRAM has been presented [14]. Those results demonstrate a two order-of-magnitude improvement over MESFET soft error rates.

\section{EXPERIMENTAL}

The heavy ion experiments were performed at the Naval Research Laboratory ion-microbeam facility, which has been described in detail elsewhere [15]. The ion beam, which is typically $\sim 1 \mathrm{~mm}$ in diameter, is diffused by a thin scattering foil, and passed through a collimating aperture and a $2.5 \mu \mathrm{m}$ pinhole that is located directly in front of the device under test (DUT). The pinhole is suspended from an $x-y$ translation stage permitting precise control over the position of ion strikes on the surface of the device with a spot size on the order of $3 \mu \mathrm{m}$.

For the optical experiments, the DUT is excited with the output of a synchronouslypumped, cavity-dumped dual-jet dye laser that is capable of producing optical pulses with durations ranging from $50 \mathrm{fs}$ to greater than 10 ps with energies of up to $50 \mathrm{~nJ}$. Cavity-dumped operation is essential in the present experiments to lower the laser pulse repetition rate and eliminate device charging effects that can arise 
as a result of longer-lived transients. For the experiments reported here, 1 ps duration optical pulses centered at $\sim 620 \mathrm{~nm}(\sim 2 \mathrm{eV})$ with a pulse repetition rate of $12 \mathrm{KHz}$ (appx. $83 \mu \mathrm{s}$ between pulses) and pulse energies of $10 \mathrm{fJ}$ to 1 pJ were used. The laser pulses are focused onto the DUT with a $150 \times$ microscope objective (Olympus MD Plan 150, numerical aperture, 0.95 ), resulting in a spot size of $\sim 1 \mu \mathrm{m}$. This is a factor of three improvement over our previous setup [5], permitting the laser spot to be focused directly onto the semiconductor material with no significant interference from metal. The apparatus is designed so that the laser-pulse autocorrelation function can be monitored continuously, providing pulse-width, pulse-shape, and laser stability information. The pulse energy is measured for each run with a calibrated, large-area photodiode.

The absorption coefficient for GaAs at 620 $\mathrm{nm}$ at $298 \mathrm{~K}$ is $4.8 \times 10^{4} \mathrm{~cm}^{-1}$ [16], giving rise to a $1 / \mathrm{e}$ absorption depth (skin depth) of $0.21 \mu \mathrm{m}$. The GaAs absorption spectrum in the vicinity of $2.0 \mathrm{eV}$ is featureless, varying by approximately $12 \%$ between 610 and $630 \mathrm{~nm}$, with a variation of $\sim 0.5 \%$ over the $1 \mathrm{~nm}$ bandwidth of the picosecond laser pulse. The reflection coefficient for $\mathrm{GaAs}$ under these conditions is 0.36 [17]; all laser pulse energies reported here are corrected for the surface reflection of GaAs using this parameter and, therefore, represent the energy absorbed by the device.

For both the ion and laser experiments the HBT under test is mounted in a high-frequency $(50 \mathrm{GHz})$ microwave package, with the emitter grounded and the base and collector wire-bonded to microstrip transmission lines. The MESFETs are mounted in a similar fashion with the source grounded and the gate and drain wire-bonded to transmission lines. Care is taken to minimize stray inductances (e.g., bond wire lengths) in order to produce transients free of artifactual ringing. The transient signals are launched from the microstrip lines onto $50 \mathrm{GHz}, 2.4 \mathrm{~mm}$ coaxial cable, passed through a bias tee, into the input of a HYPRES PSP-750 $70 \mathrm{GHz}$ superconducting sampling oscilloscope. In the microbeam experiments an additional length ( $\sim 6$ inches) of coax is required to pass through the wall of the vacuum chamber, giving rise to a slight decrease in the effective instrumental bandwidth when compared to the laser experiments.

The $1.2 \mu \mathrm{m}$ gate length GaAs MESFETs of this study are similar to those reported previously $[4,5]$, but the time-integrated charge collection measurements showed a stronger dependence on gate bias than was observed for some earlier devices [9]. The devices were fabricated by Vitesse Semiconductor as standard electrical test structures for parametric analysis of their enhancement/depletion process using ion implantation into uncompensated semi-insulating material with a $\mathrm{p}^{-}$implant for threshold control, $\mathbf{n}$ implants for source, drain and channel, and a self-aligned Schottky barrier gate. The Vitesse $1.2 \mu \mathrm{m}$ process gives rise to actual gate lengths of approximately $0.8 \mu \mathrm{m}$.

\section{ION-INDUCED TRANSIENTS}

Figure 2 shows typical $3.0 \mathrm{MeV} \alpha$-particle induced charge-collection transients measured at the collector of three different HBT devices for the three different vendors (LET of 0.5 $\mathrm{MeV} / \mathrm{mg} / \mathrm{cm}^{2}$; range of $9.2 \mu \mathrm{m}$ ). The labels $\mathrm{A}$, $B$, and $C$ identify devices from the different vendors and will be referred to as HBT-A, HBT$B$ and HBT-C in what follows. The characteristics of device A have been described elsewhere [18]. The devices were biased as indicated in the figure caption. The transients for the three devices are quite similar, consisting

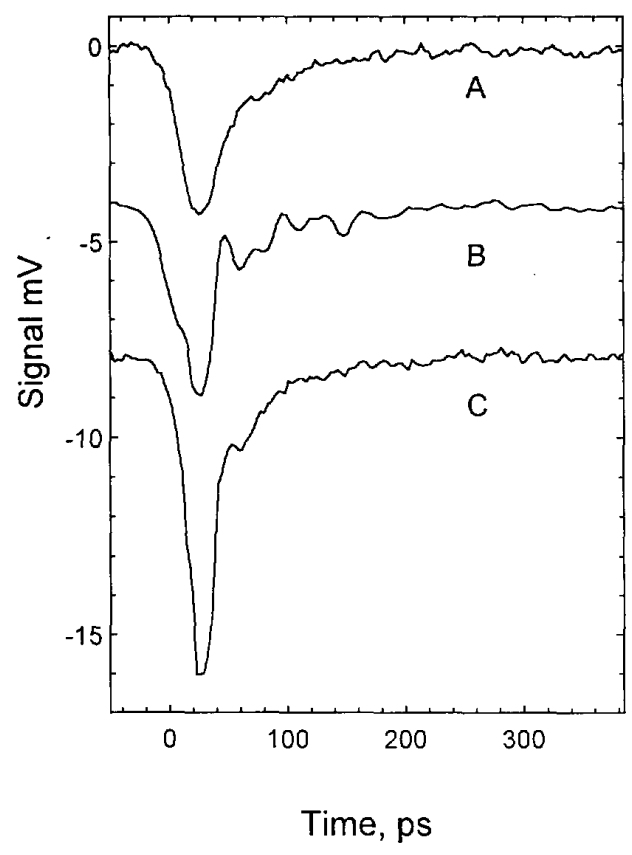

Figure 2. Collector charge-collection transients induced by $3.0 \mathrm{MeV}{ }^{4} \mathrm{He}$ for HBTs from three different vendors, labeled $\mathrm{A}, \mathrm{B}$, and $\mathrm{C}$ with the following bias conditions: (A) $V_{C E}=1.1 \mathrm{~V} ; \quad V_{B E}=1.1 \mathrm{~V} ; \quad$ (B) $V_{C E}=2.0 \mathrm{~V}$; $\mathrm{V}_{\mathrm{BE}}=0.29 \mathrm{~V}$; and (C) $\mathrm{V}_{\mathrm{CE}}=1.0 \mathrm{~V} ; \mathrm{V}_{\mathrm{BE}}=1.1 \mathrm{~V}$. The curves are offset for clairity. 
primarily of a fast, asymmetric response with a slower decay on the trailing edge. For each HBT investigated the charge collection event is complete within $250 \mathrm{ps}$ of the ionizing event. The transient for HBT-B exhibits some ringing which can be attributed to the stray inductance introduced by the significantly longer bond wires used in the packaging of that device. For both $\alpha$-particle and picosecond laser irradiation HBT$\mathrm{C}$ exhibited transients that were typically a factor of two larger than for the other devices investigated.

The differences between the HBT and MESFET charge-collection transients are illustrated by the data of fig. 3. This figure shows a

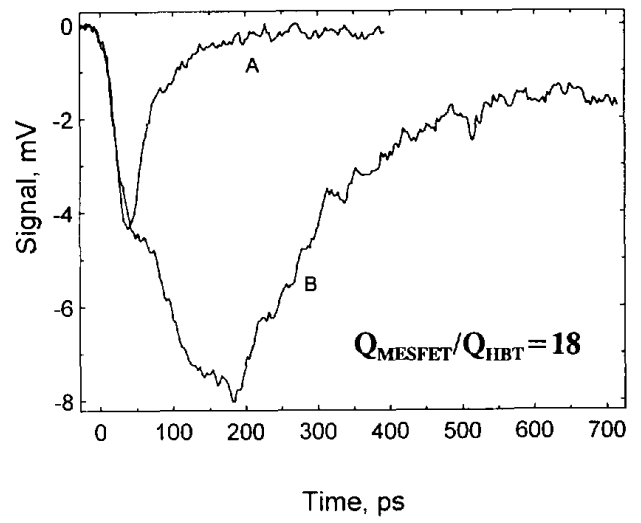

Figure 3. Comparison of collector charge-collection transients measured for HBT-A $\left(V_{C E}=1.1 \mathrm{~V} ; V_{B E}=1.1 \mathrm{~V}\right)$ and a Vitesse e-MESFET biased near pinch off $\left(V_{D}=\right.$ $2.0 \mathrm{~V} ; \mathrm{V}_{\mathrm{G}}=0 \mathrm{~V}$ ) for $3.0 \mathrm{MeV}{ }^{4} \mathrm{He}$ excitation. The ratio $\mathrm{Q}_{\mathrm{MESFET}} / \mathrm{Q}_{\mathrm{HBT}}$ is calculated from the areas under the curves with the MESFET transient extrapolated beyond 700 ps using a $2.0 \mathrm{~ns}$ relaxation time.

comparison of HBT and MESFET transients induced by $3.0 \mathrm{MeV} \alpha$-particle irradiation. The MESFET in this case is a $1.2 \mu \mathrm{m} \times 50 \mu \mathrm{m}$ enhancement-mode device, biased with $V_{D S}$ $=2.0 \mathrm{~V}$ and $\mathrm{V}_{\mathrm{GS}}=0 \mathrm{~V}$. The HBT is type $\mathrm{A}$ and is biased with $\mathrm{V}_{\mathrm{CE}}=1.1 \mathrm{~V}$ and $\mathrm{V}_{\mathrm{BE}}=1.0$ $\mathrm{V}$. In GaAs MESFETs, the largest chargecollection transients (and greatest susceptibility to SEUs) occur when biased near pinchoff. This is the case for the transient of fig. 3. As is evident, the MESFET transient is significantly larger than that of the HBT, with 8.5 times more charge collected for the MESFET for the $700 \mathrm{ps}$ window of the experiment. Because the MESFET transient clearly does not go to zero, however, this quantity represents a significant under-estimation of the total charge for the MESFET. Independent measurements [19] have determined that the relaxation time of the slower component of the MESFET chargecollection transient is on the order of 2 ns to 5 ns. Using the more conservative 2 ns value and integrating to $t=\infty$ gives a value for the ratio of collected charge for the two devices ( $\mathrm{Q}_{\text {MESFET }} / \mathrm{Q}_{\mathrm{HBT}}$ ) of 18 , with $\sim 50 \%$ of the MESFET charge collected within the first 700 ps. We note that this calculation does not take into account the presence of traps which can give rise to transients that persist for microseconds [6]. For devices biased more "on" than that of fig. 3 (up to $0.05 \mathrm{~V}$ above threshold) we have observed values for $Q_{\text {MESFET }} / Q_{H B T}$ as high as 28 .

The MESFETs of this study exhibit charge collection in excess of that deposited by the ion. The transient of fig. 3 reflects a charge enhancement of $\sim 15 \%$ using the $2.0 \mathrm{~ns}$ extrapolation. This value represents a lower limit. A charge enhancement of $>100 \%$ is obtained for timeintegrated charge-sensitive preamplifier measurements (vide infra), suggesting that $2.0 \mathrm{~ns}$ underestimates the hole recombination time in the $p$ layer. The HBT-A transient of fig. 3 , in contrast, exhibits a charge-collection efficiency of 0.07. Assuming the absence of charge enhancement mechanisms, this corresponds to a chargecollection depth of $\sim 1.3 \mu \mathrm{m}$ for the HBT.

Experimental data $[1,2,4,5]$ and computer simulation results $[5,8]$ suggest that the slower contributions (excluding traps) to the MESFET transients are a consequence of channel modulation effects that arise from excess holes located in the low-field regions below the channel (for the present device, in the potential well resulting from the buried p-layer). Near pinch-off, small modulations of the fields in the channel can give rise to large changes in the channel conduction. This effect is believed to be a primary cause of the excess charge collection that is observed in GaAs MESFETs. Correspondingly, the ratio $Q_{M E S F E T} / Q_{H B T}$ depends on the MESFET gate bias in a sensitive fashion. The charge enhancement effects in MESFETs can be reduced or eliminated by biasing the device more strongly "off", as is demonstrated in fig. 4 . In fig. 4 the MESFET is biased with $\mathrm{V}_{\mathrm{DS}}=2.0 \mathrm{~V}$ and $\mathrm{V}_{\mathrm{GS}}=-1 \mathrm{~V}$, and the channel is strongly pinched off. For the MESFET biased in this manner, the charge collection efficiency is reduced by a factor of $\sim 7$ and the ratio $\mathrm{Q}_{\mathrm{MESFET}} / \mathrm{Q}_{\mathrm{HBT}}$ is reduced to 2.5 . Unfortunate$1 y$, the bias conditions of fig. 3 , rather than fig. 4 , are typical of e-mode MESFETs in digital logic circuits. It should be noted, however, that the charge-collection characteristics can vary 


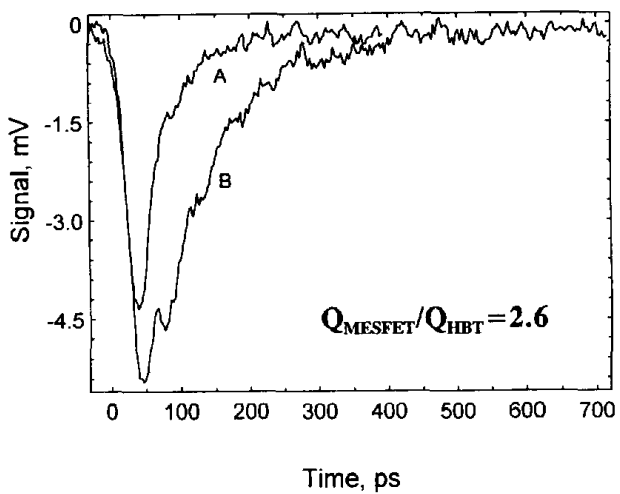

Figure 4. Comparison of collector charge-collection transients measured for HBT-A $\left(V_{C E}=1.1 \mathrm{~V} ; V_{B E}=1.1 \mathrm{~V}\right)$ and a Vitesse e-MESFET biased strongly "off" $\left(V_{D}=\right.$ $2.0 \mathrm{~V} ; \mathrm{V}_{\mathrm{G}}=-1.0 \mathrm{~V}$ ) for $3.0 \mathrm{MeV}{ }^{4} \mathrm{He}$ excitation.

significantly from device to device, even when both are from the same wafer. Our investigations indicate that this arises primarily from variations in the pinch-off voltages of the different devices. Thus, at the standard bias condition of $\mathrm{V}_{\mathrm{GS}}=0 \mathrm{~V}$ for an e-mode MESFET, different devices are expected to exhibit different susceptibilities to single-event phenomena. One method of hardening circuits based on MESFET technology might involve shifting the thresholds and/or bias conditions so that nominally "off" transistors are more strongly off.

A limited set of time-integrated chargecollection measurements [7] was performed with a $3 \mathrm{MeV}$ He beam using charge sensitive preamplifiers connected to the base and collector of

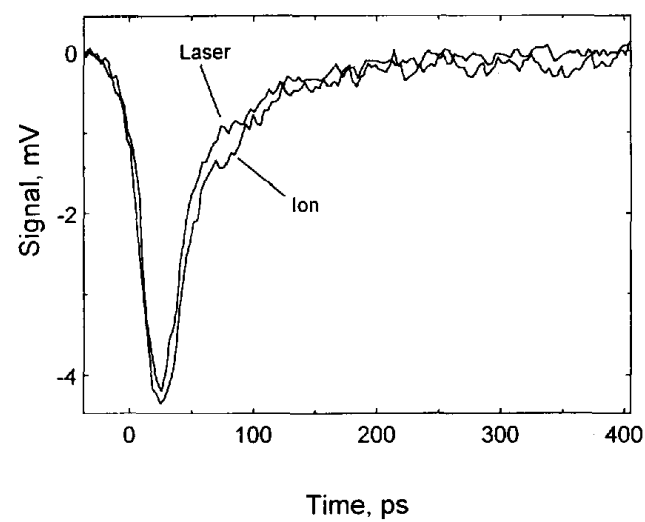

Figure 5. Comparison of charge-collection transients for HBT-A for $3.0 \mathrm{MeV}{ }^{4} \mathrm{He}$ and $19 \mathrm{fJ}$ laser pulse excitation. The bias conditions for the two curves are the same $\left(V_{C E}=1.1 \mathrm{~V} ; V_{B E}=1.0 \mathrm{~V}\right)$. No normalization factors are used. the HBT with the emitter grounded. Charge collection at the base was observed, but it was so small that it could not be measured quantitatively because of interference from noise. Charge collection spectra measured at the collector were similar to those measured by Yaktieen et al. [10] across the emitter-base junction using $4.4 \mathrm{MeV}{ }^{4} \mathrm{He}$ in that two peaks were observed with the weak high-energy peak corresponding to about 3 or 4 times the collected charge of the stronger peak. In the current study, the maximum amount of charge collected corresponds to about $0.7 \mathrm{MeV}$ and was obtained with the base biased at about $0.7 \mathrm{~V}$ and with collector bias voltages ranging from $3.3 \mathrm{~V}$ to 4.1

V. Similar measurements on MESFETs reveal $Q_{\text {MESFET }} / Q_{\text {HBT }}$ ratios consistent with those obtained from the transient measurements described above.

\section{ION-LASER COMPARISON}

Recent work has demonstrated that aboveband-gap picosecond laser excitation can be a valuable tool for investigating charge-collection dynamics and SEU phenomena in GaAs semiconductor devices $[4,5,20]$. Because of the absence of radiation damage effects, the laserbased method has proven to be an invaluable tool for the investigation of the charge-collection mechanisms of MESFETs. In this section we present evidence that laser techniques are also useful for investigations on GaAs HBTs.

Figure 5 shows a comparison of transients produced by picosecond laser pulses and 3.0 $\mathrm{MeV} \alpha$ particles on HBT-A. The laser spot was focused in the gap between the base and emitter electrodes and, therefore, charge is deposited directly in the base and collector regions (cf., fig. 1). As is evident, the two transients of fig. 5 are nearly indistinguishable, suggesting the validity of the laser technique for investigating charge-collection phenomena in GaAs HBTs. This result is not surprising given the vertical nature of the charge transport in the HBTs, and the very thin active regions of those devices. To obtain this figure the laser data was choosen to match the amplitude of the ion data which, for this device and bias conditions, corresponds to excitation by an $19 \mathrm{fJ}$ laser pulse. Both transients exhibit a slow decay component with a time constant of $\sim 100$ ps. A recent investigation into the laser induced dynamics of InP/ GaInAs/InP HBTs [12] revealed a similar $\sim 100$ ps relaxation that was attributed to the diffusion of holes. A similar mechanism may be applicable in the present case as well. 
Figure 6 shows a comparison of laser-induced charge-collection transients for the same HBT and MESFET used to generate the data of figs. 3 and 4. Bias conditions for the two curves are

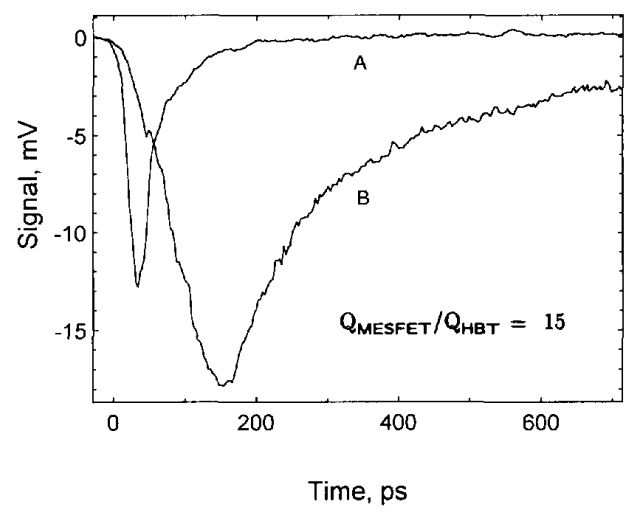

Figure 6. Comparison of charge-collection transients measured for HBT-A $\left(V_{C E}=1.1 \mathrm{~V} ; V_{B E}=1.1 \mathrm{~V}\right)$ and a Vitesse e-MESFET biased near pinch off $\left(V_{D}=1.0 \mathrm{~V}\right.$; $\mathrm{V}_{\mathrm{G}}=0 \mathrm{~V}$ ) for $74 \mathrm{fJ}, 620 \mathrm{~nm}$ laser pulses. The ratio $\mathrm{Q}_{\text {MESFET }} / \mathrm{Q}_{\mathrm{HBT}}$ is calculated from the areas under the curves with the MESFET transient extrapolated beyond 700 ps using a 2.0 ns relaxation time.

similar (but not identical) to those of fig. 3 . The two transients were produced using the same laser pulse energy (74 fJ) and spot size $(\sim 1 \mu \mathrm{m})$, with the laser spot position choosen for each device to generate the largest signal. The transients of fig. 6 are qualitatively very similar to those of fig. 3. The ratio $Q_{M E S F E T} / Q_{H B T}$ for these curves is 15 , in good agreement with the value of 18 determined for $\alpha$-particle excitation. This result supports our assertion that aboveband-gap picosecond laser pulses provide a reasonable simulation of heavy-ion induced transients.

It is well known that the charge profile created in $\mathrm{GaAs}$ is different for a $3 \mathrm{MeV} \alpha$ particle and for a $2.0 \mathrm{eV}$ laser pulse. For laser excitation the carrier density decreases exponentially with depth beneath the surface of the device whereas, for an ion, the carrier density varies with the ion's linear energy transfer (LET) as it passes through the semiconductor. Despite these very significant differences in charge deposition, the agreement between laser and ion results for the devices investigated can be rationalized by recognizing the thin, planar nature of high-frequency transistors, and that charge deposited deep into the substrate plays only a minor role in the charge-collection process. A quantitative difference between the two, however, is evident in the charge collection efficiencies measured for the two excitation methods. For both the MESFET and HBT the charge-collection efficiency (charge collected/ charge deposited times 100) for laser excitation is approximately five times that for $\alpha$-particle irradiation. This result is reasonable when it is recognized that the laser pulse deposits five times as much charge as the ion in the top 0.75 $\mu \mathrm{m}$. In future work we will examine the chargecollection efficiency as a function of laser wavelength to establish a quantitative relationship between the laser and ion experiments.

We also have examined the bias dependence of the HBT charge-collection transients for both laser and $\alpha$-particle excitation. Laser experiments have the advantage that radiationdamage effects are absent and, thus, more extensive measurements can be performed. Figure 7 shows the dependence of HBT-A on base voltage for fixed collector bias for excitation by $19 \mathrm{fJ}$ laser pulses. In contrast to the behavior of MESFETs, the HBTs of this study exhibited very little dependence on bias, with the transients of fig. 7 being very nearly indistinguishable. Limited bias dependent experiments have been performed for $\alpha$-particle irradiation with similar results. If any trend is present in the data of fig. 7 , it is that the current pulses narrow slightly and decrease in amplitude as the device is turned more on. Experiments also were

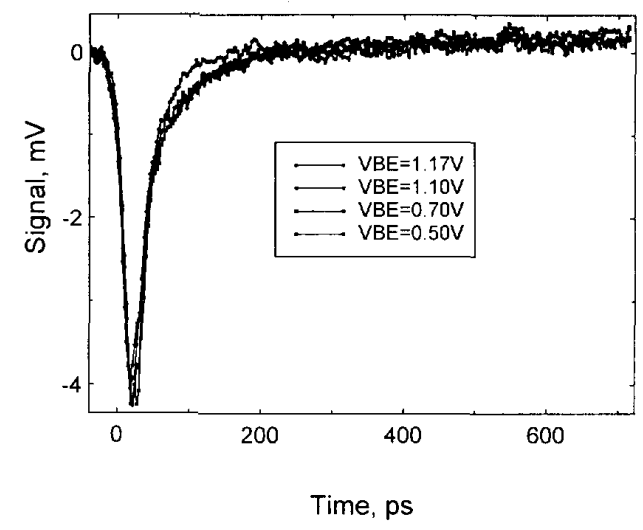

Figure 7. Dependence of collector charge-collection transients on base bias for excitation by $19 \mathrm{fJ}$ laser pulses.

performed as a function of collector bias for $\mathrm{V}_{\mathrm{CE}}$ ranging from $0.5 \mathrm{~V}$ to $5.0 \mathrm{~V}$ for both the laser and ion case. The transients exhibit very little change in shape over this range, however, the signal amplitude varies by a factor of 2 . 


\section{POSITION DEPENDENCE}

A powerful advantage of the laser method is the ability to determine the position dependence of the exciting event. Figure 8 shows a schematic diagram of the top view of an HBT device that is representative of those used in this study.

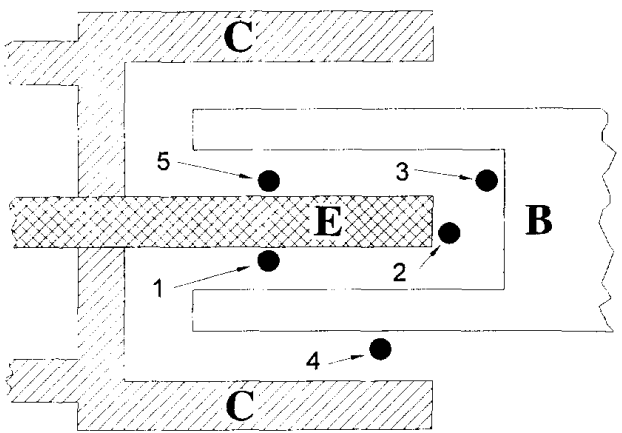

Figure 8. Schematic diagram of the top view of an HBT device showing positions of laser spots investigated. The shaded areas are metal: E-emitter metal; B-base metal; and C-collector metal.

The metal areas are indicated by the shaded regions; all other surfaces are semiconductor and are accessible to optical excitation. This figure can be used in conjunction with fig. 1 to develop a three-dimensional picture of the device.

We have probed the HBT devices in each of the positions indicated in fig. 8; some results for HBT-C are presented in fig. 9. The largest collector signals were obtained when the laser spot was positioned between the base and emitter electrodes with the spot adjacent to the

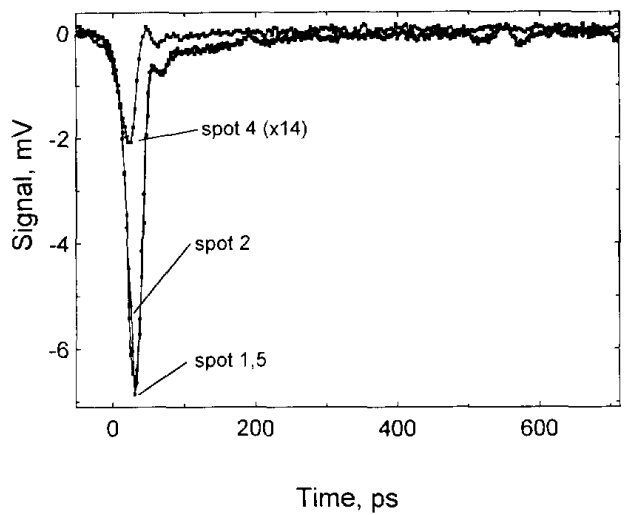

Figure 9. Charge-collection transients on the collector of HBT-C as a function of laser spot position ( $c f$. , fig. 8). Transients for spots 1 and 2 were generated with $37 \mathrm{fJ}$ laser pulse energies. $520 \mathrm{fJ}$ pulses were required to generate a detectable signal at spot 4 . emitter metal (spots 1, 2, and 5). Spots 1, 2, and 5 produced transients of comparable amplitude and nearly indistinguishable shape. Excitation at these positions deposits charge directly into the base and collector of the transistor (cf., fig. 1). Similar results are obtained for HBT-A. For excitation at spot 3 , adjacent to the base metal, the signal amplitude decreased by about $50 \%$. In general, the signal amplitude is observed to decrease as the distance from the emitter metal is increased.

Laser excitation also was provided between the base and collector electrodes (spot 4), which deposits charge directly into the collector. Using the same $37 \mathrm{fJ}$ laser pulse energy, the signal amplitude was insufficient to trigger the HYPRES (and therefore was less than $\sim 2 \mathrm{mV}$ ). The transient labeled spot 4 in fig. 9 was produced using $520 \mathrm{fJ}$ laser pulses (14 times that of the other curves), which represents the threshold for triggering the experiment at that position. These results indicate that direct excitation of the base-collector junction region results in charge collection that is approximately 45 times as efficient as excitation of the collector alone. Similar results were obtained for device A.

On the basis of these results we can conclude that the sensitive area of the typical HBT of this study is a $12 \mu \mathrm{m} \times 20 \mu \mathrm{m}$ rectangle centered on the emitter electrode and bounded by the base electrode. In addition, the positiondependent results suggest that the most sensitive node in these devices is the base-collector junction, in agreement with the conclusions of earlier workers [21]. It should be noted, however, that the emitter metal prevents direct excitation of the emitter-base junction by the laser pulse, so that a direct comparison with other earlier work $[10,11]$ is not possible.

\section{PULSE ENERGY DEPENDENCE}

Figure 10 shows the dependence of the charge-collection transient on the laser pulse energy for HBT-C. For a laser pulse of a given wavelength and pulse energy an effective LET can be calculated [20] (for example, a $37 \mathrm{fJ}$ laser pulse at $620 \mathrm{~nm}$ corresponds to an LET of 5.2 $\mathrm{MeV} / \mathrm{mg} / \mathrm{cm}^{2}$ in GaAs at the surface). Variations in pulse energy at fixed wavelength, therefore, can provide some insight into how a device might perform following interaction with higher LET ions in a space environment. The data of fig. 10 indicate that at higher laser pulse energies the collector current pulse broadens, and the peak amplitude of the current transient 


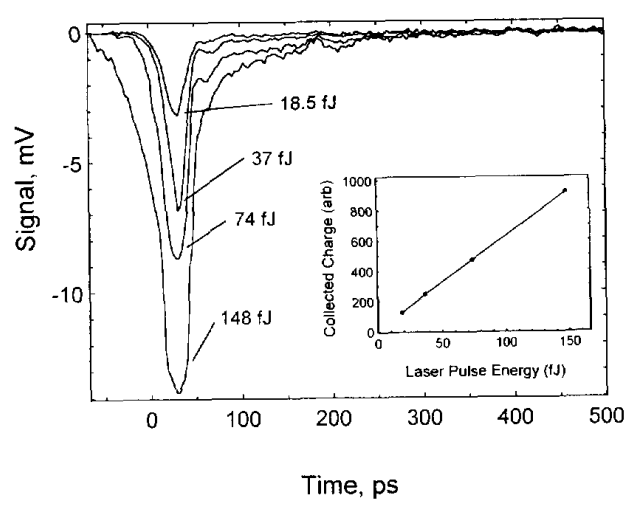

Figure 10. Laser pulse energy dependence of the collector transients for HBT-C. The inset shows the integrated area of these curves as a function of laser pulse energy.

appears to saturate. The inset of fig. 10, however, shows that the integrated charge varies in a linear fashion as a function of laser pulse energy. Thus, the charge collection efficiencies of the HBTs of this study might be expected to vary in a linear fashion with ion LET.

This behavior is in contrast to that of $\mathrm{GaAs}$ MESFETs which exhibit enhanced gain at higher pulse energies [19]: the charge enhancement mechanisms of MESFETs become more pronounced as the carrier density is increased.

\section{CLOCK-RATE EFFECTS}

The results presented here are particularly significant when considered in the light of recent results involving high speed MESFET combinational logic $[22,23]$. Those results demonstrate that combinational logic circuits are more susceptible to SEUs at higher clock frequencies because of the increased probability that an ion strike will occur within a "critical window" prior to the rising clock edge (the "critical window" is defined such that upsets will occur for sufficiently energetic ion strikes within the window, but will not occur for ion strikes outside the window). For a given energy deposition, the width of this critical window is determined by the shape and duration of the current pulse created. The GaAs MESFET circuits investigated [23] exhibited a critical window on the order of $2-5$ ns when excited near threshold. This is consistent with the $2-5$ ns time constant measured for the slower component of the charge-collection transients [19]. The HBT charge-collection transients, in contrast, decay within $250 \mathrm{ps}$ (with $90 \%$ of the charge being collected within the first $75 \mathrm{ps}$ ), and will result in a significant decrease in the width of this "critical window", leading to a corresponding decrease in the SEU error rate at high clock frequencies if all other parameters are assumed to be equal.

\section{CONCLUSIONS}

We have presented a comparison of the charge-collection dynamics of high-performance HBT and MESFET devices. These result reveal that the HBT devices exhibit very fast chargecollection pulses that are $90 \%$ complete within $75 \mathrm{ps}$ after the ionizing event. This is in contrast to the behavior of GaAs MESFETs which can exhibit (depending on the bias conditions) charge-collection transients that persist for microseconds. The fast response of the HBTs of this study is attributed to the vertical nature of charge transport in these devices, and the resultant short transit times for the carriers. In addition, the HBT devices appear insensitive to carriers deposited below the active region of the device; such carriers are largely responsible for the enhanced charge collection that is observed in GaAs MESFETs. Because of the very fast charge-collection dynamics, the HBT devices of this study exhibit charge-collection efficiencies that are up to 28 times less than those of MESFETs for identical excitation conditions. These characteristics are expected to result in significantly improved SEU performance for logic circuits and memories constructed with HBT technology.

Experiments involving above-band-gap pulsed picosecond laser excitation were performed. These studies produce transients that are very nearly indistinguishable from those produced with $\alpha$-particle irradiation, and reveal that picosecond laser pulses are a valuable tool for investigating charge-collection phenomena in these devices. Studies as a function of laser spot position reveal that the charge-collection efficiency falls off rapidly as the distance from the emitter contact is increased. This result, when coupled with the very thin collection depth noted above, suggests that the sensitive volume of these devices is quite small, in agreement with earlier investigations $[10,11]$.

The ultrafast charge collection dynamics of HBTs are particularly significant when clockrate effects are considered. GaAs MESFET circuits have exhibited enhanced SEU sensitivity when operated at high clock rates $[22,23]$. The absence of slowly relaxing tails on the HBT charge-collection transients will permit HBTbased circuits to operate at considerably higher 
clock frequencies than GaAs MESFET circuits before clock-rate effects become significant.

\section{REFERENCES}

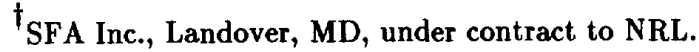

1. B.W. Hughlock, G.S. LaRue, and A.H Johnston, "Single-Event Upset in GaAs E/D MESFET Logic", IEEE Trans. Nuc. Sci. NS-37, 1894 (1990).

2. B.W. Hughlock, T. Williams, A.H Johnston and R.E. Plaag, "Ion Induced Charge Collection in GaAs MESFETs and its Effect on SEU Vulnerability", IEEE Trans. Nuc. Sci. NS-38, 1442 (1991).

3. T.R. Weatherford, L. Tran, W.J. Stapor, E.L. Peterson, J.B. Langworthy, D. McMorrow, W.G. AbdelKader, and J.P. McNulty, "Proton and Heavy Ion Upsets in GaAs MESFET Devices", IEEE Trans. Nuc. Sci. NS-38, 1450 (1991).

4. D. McMorrow, Alvin R. Knudson and A.B. Campbell, "Fast Charge Collection in GaAs MESFETs", IEEE Trans. Nuc. Sci. NS-37, 1902 (1990).

5. D. McMorrow, J.S. Melinger, A.R. Knudson, T.R. Weatherford, L.H. Tran, and W.R. Curtice, "Picosecond Charge-Collection Dynamics in GaAs MESFETs:” IEEE Trans. Nuc. Sci., NS-39 (1992).

6. S. Buchner, K. Kang, D.W. Tu, A.R. Knudson, A.B. Campbell, D. McMorrow, V. Srinivas, and Y.J. Chen, "Charge Collection in GaAs MESFETs and MODFETs", IEEE Trans. Nuc. Sci. NS-38, 1370 (1991).

7. A.B. Campbell, A. Knudson, D. McMorrow, W. Anderson, J. Roussos, S. Espy, S. Buchner, K. Kang, D. Kerns, and S. Kerns, "Ion-Induced Charge Collection in GaAs MESFETs", IEEE Trans. Nuc. Sci. NS-36, 2292 (1989).

8. T.R. Weatherford, D. McMorrow, W.R. Curtice, A.R. Knudson, and A.B. Campbell, "Single Event Induced Charge Transport Modeling of GaAs MESFETs", IEEE Trans. Nuc. Sci. NS-40 (December, 1993).

9. A.R. Knudson, A.B. Campbell, D. McMorrow, S. Buchner, K. Kang, T. Weatherford, V. Srinives, G.A. Swartzlander, Jr., and Y.J. Chen, "Pulsed Laser-Induced Charge Collection in GaAs MESFETs", IEEE Trans. Nuc. Sci., NS-37, 1909 (1990).

10. M.H. Yaktieen, P.J. McNulty, J.E. Lynch, D.R. Roth, J.F. Salzman, and J.H. Yuan, "Charge Collection and SEU Sensitivity for Ga/As Bipolar Devices", IEEE Trans. Nuc. Sci. NS-36, 2300 (1989).

11. P.J. McNulty, M.H. Yakieen, J.E. Lynch, W.M. Weber, H.T. Yuan, and J.F. Salzman, "Charge Collection in $\mathrm{HI}^{2} \mathrm{~L}$ Bipolar Transistors", IEEE Trans. Nuc. Sci. NS-35, 1613 (1988).
12. T.F. Carruthers, I.N. Duling III, O. Aina, M. Mattingly, and $M$. Serio, "Responses of $\operatorname{InP} / \mathrm{GaInAs} / \operatorname{InP}$ Heterojunction Bipolar Transistors to 1530 and $620 \mathrm{~nm}$ Ultrafast Optical Pulses", Appl. Phys. Lett. 59, 327 (1991).

13. B. Hughlock, A. Johnston, T. Williams, and J. Harrang, "A Comparison of Charge Collection Between GaAs MESFETs and III-V HFETs", IEEE Trans. Nuc. Sci. NS-39, 1642 (1992).

14. J. Cutchin, P.W. Marshall, T.R. Weatherford, J. Langworthy, E.L. Peterson, A.B. Campbell, S. Hanka and A. Peczalski, "Heavy Ion and Proton Analysis of a GaAs C-HIGFET SRAM", IEEE Trans. Nuc. Sci. NS-40 (December 1993).

15. A.R. Knudson and A.B. Campbell, "Use of an Ion Microbeam to Study Single Event Upsets in Microcircuits", IEEE Trans. Nuc. Sci. NS-28, 4017 (1981).

16. D.D. Sell and H.C. Casey, "Optical Absorption and Photoluminescence Studies of Thin GaAs Layers in GaAs- $\mathrm{Al}_{x} \mathrm{Ga}_{1-x}$ As Double Heterostructures", J. Appl. Phys. 45, 800 (1974).

17. H.R. Phillip and H. Dhrenreich, "Optical Properties of Semiconductors", Phys. Rev. 129, 1550 (1963).

18. P.M. Enquist, D.B. Slater, Jr., and J.W. Swart, "Complementary AlGaAs/GaAs HBT $\mathrm{I}^{2} \mathrm{~L}$ ( $\left.\mathrm{CHI}^{2} \mathrm{~L}\right)$ Technology", IEEE Electron Device Lett., 13, 180 (1992).

19. D. McMorrow, A.R. Knudson, A.B. Campbell, unpublished work, 1991.

20. S. Buchner, A. Knudson, K.Kang, and A.B. Campbell, "Charge Collection From Focused Picosecond Laser Pulses", IEEE Trans. Nuc. Sci. NS-35, 1517 (1988).

21. D.C. Scott, D.V. Plant, and H.R. Fetterman, “60 $\mathrm{GHz}$ Sources Using Optically Driven Heterojunction Bipolar Transistors", Appl. Phys. Lett. 61, 1 (1992).

22. R. Schneiderwind, D. Krening, S. Buchner, K. Kang, and T.R. Weatherford, "Laser Confirmation of SEU Experiments in GaAs MESFET Combinational Logic", IEEE Trans. Nuc. Sci., NS-39, 1665 (1992).

23. S. Buchner, K. Kang, D. Krening, G. Lannan, and R. Schneiderwind, "Timing Effects Associated with SingleEvent Upsets in a Logic Circuit", IEEE Trans. Nuc. Sci. NS-40 (December, 1993). 